La Revue

des Droits

de l'Homme

\section{La Revue des droits de l'homme}

Revue du Centre de recherches et d'études sur les droits fondamentaux

Actualités Droits-Libertés | 2017

\title{
Rapport annuel 2017 de la FRA : miroir d'une Union européenne en perte de vitesse sur les droits fondamentaux?
}

Agence des droits fondamentaux de l'Union européenne

Nathan Dufrois, Agathe Fadier et Elisabeth Miller

\section{OpenEdition}

Journals

Édition électronique

URL : http://journals.openedition.org/revdh/3516

DOI : $10.4000 /$ revdh.3516

ISSN : 2264-119X

Éditeur

Centre de recherches et d'études sur les droits fondamentaux

Référence électronique

Nathan Dufrois, Agathe Fadier et Elisabeth Miller, « Rapport annuel 2017 de la FRA : miroir d'une Union européenne en perte de vitesse sur les droits fondamentaux? », La Revue des droits de l'homme [En ligne], Actualités Droits-Libertés, mis en ligne le 28 novembre 2017, consulté le 19 avril 2019. URL: http://journals.openedition.org/revdh/3516 ; DOI : 10.4000/revdh.3516

Ce document a été généré automatiquement le 19 avril 2019

Tous droits réservés 


\section{Rapport annuel 2017 de la FRA : miroir d'une Union européenne en perte de vitesse sur les droits fondamentaux?}

Agence des droits fondamentaux de l'Union européenne

Nathan Dufrois, Agathe Fadier et Elisabeth Miller

1 «Le renforcement de l'architecture institutionnelle de protection des droits fondamentaux aura atteint son objectif lorsque les citoyens de l'Union européenne sentiront que leurs droits sont réellement garantis et pris en compte $»^{1}$. Tel est le bilan en demi-teinte que dresse l'Agence des droits fondamentaux de l'Union européenne (FRA) à l'issue d'une décennie de promotion des droits et libertés au sein de l'Union. Le dixième anniversaire de sa création constitue en effet une opportunité idéale afin de développer un regard rétrospectif critique sur son action ainsi que sur la place des droits fondamentaux au sein de la construction européenne. Cette réflexion, au cœur du rapport 2017 de la FRA, semble d'autant plus nécessaire que la remise en cause de la protection des droits les plus élémentaires est toujours d'actualité dans un contexte de crises multiples. La gestion des flux de réfugiés, le marasme économique dans certains pays du sud ou encore la remise en question de l'État de droit en Pologne fragilisent l'Union européenne des droits fondamentaux.

2 Créée en $2007^{2}$, la FRA succéda à l'Observatoire européen des phénomènes racistes et xénophobes (EUMC). D'après l'article 2 du règlement $n^{\circ} 168 / 2007$, l'Agence a pour objectif de fournir « aux institutions, organes, organismes et agences compétents de l'Union ainsi qu'à ses États membres lorsqu'ils mettent en œuvre le droit de l'Union européenne, une assistance et des compétences en matière de droits fondamentaux». C'est donc essentiellement une tâche d'expertise qui est confiée à la FRA, ce qui la distingue de la plupart des agences de l'Union européenne (UE) ${ }^{3}$. Elle l'effectue par la collecte de données ainsi que par la rédaction de divers rapports et communications. Au moment où la signature du Traité de Lisbonne confirmait la place essentielle des droits fondamentaux ${ }^{4}$ 
au sein du projet européen, la création de l'Agence, dotée d'une mission de veille et de surveillance, fut considérée comme un élément important de la protection des droits fondamentaux dans l'espace européen. Sa structuration, relativement originale, fut perçue comme une garantie de son indépendance ${ }^{5}$.

Cependant, une décennie plus tard, il convient de s'interroger sur la portée de l'action de la FRA dans un contexte particulièrement dégradé où les institutions de l'Union paraissent relativement impuissantes face aux crises diverses. À la lumière des évènements récents, elle-même questionne le caractère limité de son mandat et de son champ d'action qui témoigne de la frilosité des États membres ${ }^{6}$. Captive d'un statut largement restrictif qui l'empêche de pleinement dénoncer les atteintes aux droits fondamentaux ou d'avoir un impact significatif sur les textes législatifs de l'Union ${ }^{7}$, son bilan s'avère extrêmement modeste. À défaut de pouvoir jouer un véritable rôle de coordination ou même de surveillance, la FRA semble s'être cantonnée à une mission de communication, nécessaire mais insuffisante.

Le bilan décennal qui caractérise ce rapport offre ainsi une opportunité de s'interroger, au prisme de l'action de la FRA et des efforts de l'Union, sur l'efficacité de la protection des droits fondamentaux au sein de l'espace européen ${ }^{8}$. Afin de comprendre son rôle, il convient d'analyser ce que le rapport révèle du positionnement de la FRA dans son environnement institutionnel (I), avant de se demander si son manque d'ambition ne reflèterait pas, en creux, la place que les droits fondamentaux occupent actuellement dans la construction européenne (II).

\section{1/ - Le positionnement de la FRA dans le système de protection des droits fondamentaux}

5 La FRA est une organisation tenue de se positionner au sein d'un environnement complexe. Dotée de faibles prérogatives et ballotée entre différents intérêts, elle affiche un positionnement ambigu' au sein de l'environnement institutionnel de l'Union (A), qui répond à ses conditions d'existence tout en nuisant paradoxalement à sa visibilité. Hors de l'UE, elle se situe au carrefour d'une multiplicité d'acteurs nationaux, européens et internationaux de protection des droits de l'Homme (B).

\section{A/ - La FRA dans l'environnement institutionnel de l'Union : une agence dotée de faibles prérogatives et traversée par différents intérêts}

6 Tout d'abord, il convient de souligner que la FRA est limitée par son statut, qui, en la cantonnant à un rôle d'expertise et d'information, restreint son champ d'action ${ }^{10}$. Cependant, au-delà du texte, il semblerait que la FRA ait également une interprétation restrictive de ses propres statuts. Cela peut s'expliquer par le fait qu'elle essaie de ménager les différents intérêts des institutions et organes européens, intérêts qui peuvent parfois être contradictoires. Ce phénomène est perceptible lorsqu'est analysée la manière avec laquelle elle s'adresse aux différents acteurs institutionnels de l'UE.

7 Concernant les États membres, auxquels elle s'adresse à la fois en tant que pouvoir exécutif et législatif de l'UE (Parlement européen), elle adopte une attitude prudente. Le langage retenu est relativement diplomatique (utilisation du verbe «devoir» au 
conditionnel). Ainsi, dans le passage le plus "offensif» du rapport, à l'encontre de la Pologne $e^{11}$, la FRA estime que cet État "devrait prendre en considération les mécanismes européens et internationaux de surveillance des droits de l'Homme ». Lorsqu'elle aborde les sujets clivants, telle la question de l'avortement en Pologne ${ }^{12}$ ), elle expose factuellement la situation, sans prendre parti ${ }^{13}$. De manière générale, elle fait état des affaires en cours ou jugées au cours de l'année dans les États membres dans le cadre d'une approche à court terme qui empêche de mettre en exergue les évolutions, positives comme négatives de la situation dans un pays donné. En outre, il est fréquent de trouver sur un même sujet un État membre cité à deux reprises, dans le cadre d'un exemple négatif (e.g. législation contraire au droit de l'UE ou à la Convention de sauvegarde des droits de l'homme et des libertés fondamentales - Convention EDH), puis, dans le cadre d'un exemple positif (e.g. «bonnes pratiques $\left.»^{14}\right)$.

Dans son rapport, la FRA affiche en outre son soutien à la Commission européenne, soutenue à la fois dans sa fonction de garante des droits fondamentaux et de défense de l'intérêt communautaire. La FRA : valorise le travail de la Commission; encourage les États membres à transposer les textes communautaires (e.g. directive sur les droits procéduraux dans les procédures pénales ${ }^{15}$ ) ou à reprendre les négociations (e.g. directive sur la lutte contre les discriminations bloquée depuis 8 ans $^{16}$ ); propose d'utiliser les outils développés par la Commission européenne - même lorsque ceux-ci visent davantage à préserver l'intérêt communautaire que les droits fondamentaux ${ }^{17}$. Le seul domaine pour lequel le rapport pointe une déficience éventuelle de la Commission est celui des droits de l'enfant, considérant qu'elle appelle à remplacer le plan d'action de l'UE pour les mineurs non accompagnés - arrivé à échéance en 2014 - par un nouveau plan sur les enfants migrants ${ }^{18}$.

Sont également mentionnés à plusieurs reprises les travaux du Parlement européen (PE) ${ }^{19}$ , institution traditionnellement active dans le domaine des droits de l'Homme, tant sur le plan interne ${ }^{20}$ qu'externe $^{21}$. Ce dernier n'hésite pas à être offensif envers les États membres lorsqu'ils enfreignent les droits fondamentaux. C'est d'ailleurs le PE qui avait le premier évoqué la création d'une Charte autonome pour les droits fondamentaux de l'UE. Amené à se prononcer dans le cadre de la procédure de décharge budgétaire, il se « félicite des résultats positifs de l'Agence ", et salue "son approche proactive à l'égard du Parlement européen $\|^{22}$. Il n'hésite pas à « déplorer » un mandat trop restrictif qui « continue de limiter son rôle en matière d'appui aux droits fondamentaux » et appelle à un élargissement de son domaine de compétence à tous les domaines protégés en vertu de la Charte, notamment les questions de coopération policière et judiciaire ${ }^{23}$.

D'autres agences de l'UE, amenées à agir dans les domaines des droits de l'Homme, sont également mentionnées. Il s'agit d'agences en relation avec l'ancien 3ème pilier (Justice et affaires intérieures) et de l'institut pour l'Égalité entre les femmes et les hommes. Concernant les agences JAI, leur mention semble s'inscrire dans un processus d'autopromotion de la part de la FRA qui est chargée cette année de la coordination du réseau des agences liées aux problématiques migratoires. En effet, avec la question des enfants migrants - à laquelle la FRA consacre son forum annuel et qui est traitée de manière transversale dans le rapport annuel -, l'agence tente de s'affirmer dans l'environnement institutionnel des acteurs des politiques d'asile et d'immigration de l'UE, alors même qu'elle est dotée de pouvoirs moins importants que des agences comme Frontex ou Europol. 
11 Enfin, une attention particulière est portée à la société civile. À plusieurs reprises dans le rapport, la FRA s'affiche comme le porte-voix des ONG de défense des droits de l'Homme. De manière plus étonnante, elle cite les entreprises, dans le cadre d'un exemple de bonnes pratiques à travers des partenariats publics-privés pour lutter contre la pauvreté des enfants. Peut-être faut-il voir dans le renforcement de l'implication de la société civile la volonté de la FRA de renforcer son « soft power » face aux difficultés d'exercer un véritable rôle de surveillance.

12 Les difficultés de lecture du positionnement de la FRA sont susceptibles de trouver plusieurs explications. Au-delà de la limitation statutaire, la FRA ne peut pas, diplomatiquement, se montrer trop offensive envers les États membres ou les institutions de l'UE. Ces dernières détiennent en effet un pouvoir direct sur l'agence, notamment en votant son budget et en lui octroyant la décharge ${ }^{24}$. Une autre explication stratégique pourrait être que le ton mesuré de son rapport lui permet d'influencer plus efficacement les États membres. Si cette diplomatie des experts a permis à la FRA d'élargir son champ de compétence à de nouveaux domaines (e.g. handicap), celle-ci possède cependant des limites. Malgré les exhortations du Parlement européen en ce sens, il apparaît en effet peu probable que le Conseil élargisse le mandat de l'Agence à des domaines d'action comme la coopération policière et judiciaire.

Si la FRA effectue une forme de surveillance généralisée des institutions et organes de l'UE, son pouvoir n'est en aucun cas contraignant pour les différents acteurs. Tout au plus affiche-t-elle timidement son rôle de témoin des violations des droits fondamentaux ${ }^{25}$. C'est d'ailleurs davantage sur son rôle de diffusion de l'information et de communication entre acteurs de l'UE qu'elle semble vouloir insister à travers ce rapport. Reste alors à déterminer quelles sont ses relations qu'elle entretien avec les acteurs extracommunautaires de protection des droits de l'Homme, tant nationaux, européens, qu'internationaux?

\section{B/ - La FRA dans l'environnement national, européen et international des droits de l'Homme : collaborateur ou communicant?}

14 La FRA s'apparente à un organe central de coopération avec les autres acteurs extracommunautaires de la protection des droits de l'Homme, aussi bien au niveau national, européen qu'international. Il est possible d'envisager cette position tant d'un point de vue formel, en analysant le statut de l'Agence (i), que d'un point de vue matériel, à la lecture du rapport annuel sur les droits fondamentaux (ii).

\section{i. La FRA et les autres acteurs : au carrefour entre la protection nationale et internationale des droits de l'Homme}

15 L'article 2 du règlement ${ }^{26}$ créant la FRA précise que l'Agence « a pour objectif de fournir aux institutions, organes, organismes et agences compétents de l'Union ainsi qu'à ses États membres lorsqu'ils mettent en oeuvre le droit de l'Union Européenne, une assistance et des compétences en matière de droits fondamentaux afin de les aider à respecter pleinement ces derniers". À cette fin, elle remplit des missions d'observation et de recueil de données. Néanmoins, il convient de préciser que ses conclusions, avis et rapports « ne peuvent porter sur des propositions de la Commission (...) du traité ou des positions prises par les institutions dans le cadre de procédures législatives que lorsqu'une demande a été présentée par l'institution concernée (...). Ils ne portent 
pas sur la légalité des actes (...) ni sur la question de savoir si un État membre a manqué à une des obligations qui lui incombent en vertu du traité ». Si on la compare aux autres institutions nationales, européennes et internationales, l'Agence bénéficie donc de moyens de surveillance limités, notamment en comparaison de sa mission de coopération, beaucoup plus développée ${ }^{27}$. Toutefois, cela ne l'empêche pas de se positionner au même niveau que ces dernières. En effet, malgré la faiblesse de ses pouvoirs, elle tente d'affirmer sa position de carrefour auprès d'institutions aux prérogatives nettement plus étendues que les siennes.

16 Tout d'abord, la FRA collabore avec le Conseil de l'Europe ${ }^{28}$. Cette organisation régionale est un acteur primordial dans le domaine de la protection des droits de l'Homme. Considérant la présence d'un représentant de cette institution au sein du Conseil d'Administration de la FRA, il serait légitime de s'attendre à une importante coopération formelle ${ }^{29}$. Cependant, au sein du rapport, l'Agence mentionne peu son travail de coopération avec le Conseil de l'Europe. Elle ne s'étend pas non plus sur le travail des différents organes strasbourgeois et ne les met pas en valeur comme elle peut le faire pour les institutions de l'Union et pour les États membres. Ce constat témoigne de la difficile coordination de ces deux organisations régionales ${ }^{30}$.

De la même façon, elle évoque peu les acteurs nationaux de défense des droits de l'Homme. Certes, elle rappelle dans son rapport annuel les affaires en cours ou jugées devant les juridictions nationales ainsi que les «bonnes pratiques». Cependant, elle ne revient pas toujours sur le travail des organismes indépendants des États membres, chargés de la défense des droits et libertés. Il convient de souligner que, comparativement, les prérogatives de la FRA sont limitées face aux institutions nationales et indépendantes comme le Défenseur des droits ${ }^{31}$.

Enfin, la FRA ne peut pas non plus s'affirmer comme un organe de protection des droits de l'Homme tel que le Comité des droits de l'Homme des Nations Unies, organe composé d'experts indépendants surveillant la mise en œuvre du Pacte international relatif aux droits civils et politiques ainsi que de ses protocoles. Ce dernier est devenu un organe quasi-judiciaire. Tous les États parties sont en effet tenus de lui présenter, à intervalles réguliers, généralement tous les quatre ans, des rapports sur la mise en œuvre des droits consacrés par le Pacte. Le Comité examine chaque rapport et fait part de ses préoccupations et de ses recommandations à l'État partie sous la forme d' "observations finales» où sont analysées dans le détail les obligations de fond et de procédure qui incombent aux États. Le Comité peut se prononcer sur le fond de l'affaire et constater une violation du Pacte par un État parti. Ces constatations « présentent certaines caractéristiques principales d'une décision judiciaire $\aleph^{32}$. Ainsi, la FRA, bien que coopérant avec les comités onusiens, n'a pas les mêmes caractéristiques et compétences que ces derniers, notamment, au regard de leur fonction quasi-judiciaire issue tant de leurs statuts que de leur pratiques ${ }^{33}$.

19 En dépit du constat de la position centrale de la FRA, reflétée par sa mission de coopération, force est de constater que ses pouvoirs sont en décalage vis-à-vis des autres institutions avec lesquelles elle collabore. En réaction, l'Agence aurait pu développer sa fonction de surveillance visant à faire respecter les obligations incombant aux États membres tirées de la Charte des droits fondamentaux de l'Union européenne. Au vu des dispositions du règlement qui l'a instaurée, il parait clair que là n'était pas l'objectif des États membres. Pour autant, elle n'aurait pas été la première organisation à outrepasser 
ses compétences ${ }^{34}$ ayant recours pour ce faire aux outils d'expertise à sa disposition et notamment à son rapport annuel.

Reste maintenant à s'interroger sur l'effectivité du rôle de la FRA. À la lecture du rapport, il est possible de se demander si la position de l'Agence est le reflet d'une volonté de trouver sa place parmi les institutions spécialisées dans la protection des droits de l'Homme ou bien le moyen d'élargir le spectre «droits fondamentaux» de l'Union européenne.

\section{ii. Une position affichée de " collaborateur » : « trouver sa place » ou développer la protection des droits fondamentaux de l'Union européenne?}

21 La FRA utilise sa position de collaboratrice au-delà de ses statuts pour élargir ses compétences et, par ricochet, pour mettre en valeur un spectre élargi d'action de l'UE en matière de droits fondamentaux. À la lecture du rapport annuel, il ressort que la FRA valorise son rôle d'extension des activités de l'UE en matière de protection des droits fondamentaux. Cela apparait notamment dans trois domaines distincts : la lutte contre les discriminations, le droit des enfants et le handicap. À noter que la stratégie de la FRA varie selon le niveau de compétence et d'ancienneté d'action de l'UE dans chacun des domaines.

La lutte contre les discriminations constitue un domaine de prédilection de l'Union et de la FRA. C'est d'ailleurs traditionnellement le premier domaine abordé par le rapport annuel après le chapitre consacré à la Charte. L'Agence qui a remplacé l'observatoire européen des phénomènes racistes et xénophobes depuis sa création en 2007 a, de par son histoire, une expertise plus poussée sur ces questions ${ }^{35}$. La FRA valorise le travail de l'UE dans le domaine de la lutte contre les discriminations et intervient en soutien de la Commission européenne et du Parlement européen en appelant les États membres à adopter la directive de 2008, dont les négociations sont bloquées depuis 8 ans. Dans les domaines où elle joue un rôle fort, où elle peut être plus protectrice et plus innovante au regard de son histoire, la FRA favorise ainsi les mesures prises au niveau de l'UE.

À l'opposé, s'agissant de la protection des droits des personnes handicapées, la FRA encourage vivement les États membres et les institutions de l'UE à exécuter les dispositions contenues dans la Convention relative aux droits des personnes handicapées (CDPH) adoptée le 13 décembre 2016 par l'Assemblée générale des Nations Unies et à laquelle l'UE est partie ${ }^{36}$. Elle recommande de prêter attention aux observations générales du comité en charge de l'application de cette convention, même si elles ne sont pas juridiquement contraignantes et cite ses travaux. Elle se positionne alors en tant que simple intermédiaire entre l'UE, les États membres et d'autres organisations internationales, ici l'ONU et, plus précisément, le Comité des droits des personnes handicapées (CRPD). Le droit des personnes handicapées étant un domaine récent d'intervention, la FRA s'en remet aux travaux d'autres organisations internationales. Elle reste donc dans une sorte de «zone de confort » quand elle traite d'un domaine qui ne fait pas forcément consensus parmi les États membres, souvent réticents à confier plus de compétences à l'UE. Par ailleurs, l'Agence insiste sur les efforts entrepris par les institutions de l'UE pour mettre en œuvre la convention et les observations du comité. S'il s'agit de montrer que l'UE met tout en œuvre pour respecter ses obligations découlant de la ratification de la Convention, il est possible de se demander si la FRA n'utilise pas aussi cette partie du rapport pour affirmer et promouvoir le nouveau rôle de l'UE dans un 
nouveau domaine des droits et libertés, quand bien même il s'agit d'un domaine où ses compétences sont limitées.

La manière dont la FRA traite le sujet des droits de l'enfant dans son rapport annuel est également révélatrice de la stratégie mise en œuvre par les institutions européennes pour s'affirmer en tant qu'acteur de la protection internationale des droits et libertés. En effet, alors que les droits de l'enfant sont protégés au niveau des Nations Unies avec la Convention relative aux droits de l'enfant adoptée par l'Assemblée générale le 20 novembre 1989, l'UE a également décidé de se saisir de cette question. Dans son rapport, la FRA énonce tour à tour l'ensemble des outils à disposition de l'UE pour garantir les droits des enfants de manière transversale. Ainsi, elle commence par évoquer la pauvreté des enfants dans le cadre de la Stratégie UE 2020, en mentionnant également le Semestre européen et le pilier européen des droits sociaux. Puis, elle traite de l'accès à la justice en mentionnant la directive sur les garanties procédurales en faveur des enfants soupçonnés ou auteurs d'infractions pénales. Enfin, elle insiste sur la question des enfants migrants, thème également abordé dans la partie relative à l'asile et à l'immigration, en appelant la Commission européenne et les États membres à adopter un nouveau plan d'action. La diversité des outils et des actions de l'UE sur les droits des enfants rapportée par l'Agence reflète la dynamique dans laquelle se trouve l'UE au regard des droits fondamentaux. Entre récupération et légifération, elle semble vouloir, étape par étape, conquérir son rang au sein des acteurs de protection des droits de l'Homme avec comme porte-parole la FRA.

Malgré tout, l'Agence des droits fondamentaux de l'Union Européenne semble limitée dans son rôle d'acteur de la protection des droits et libertés. En dépit de sa position de carrefour et de son rôle de collaboratrice, elle apparait à la lecture du rapport davantage cantonnée à un rôle de publicité vis-à-vis des mesures de l'UE. Sa place reste en conséquence mineure, réduite à un simple rôle d'organe de communication. Outre les limitations liées à son statut ${ }^{37}$, il est possible se demander si cette situation ne serait pas le reflet de la difficulté de l'UE à développer une protection effective des droits et libertés.

\section{2/ - Un rapport significatif de la difficulté de l'Union européenne à développer une protection effective des droits fondamentaux}

L'analyse menée jusqu'à présent a pu montrer que la FRA, malgré ses prétentions initiales, ne possède qu'un faible rôle en termes de coordination des institutions de protection des droits fondamentaux. Par ailleurs, en raison de son statut, ses capacités de surveillance et de dénonciation demeurent insuffisantes. Elle assure avant tout une mission de communication qui invite à réinterroger sa relation avec le Conseil de l'Europe (A). Enfin, il est possible de se demander si le défaut d'ambition de la FRA ne serait finalement pas le reflet de la place que les droits fondamentaux occupent actuellement dans la construction européenne (B).

\section{A/ - Une volonté de communiquer}

Ce rapport 2017, comme les précédents, témoigne d'une sous-utilisation de la Charte des droits fondamentaux aux niveaux étatique et supra-étatique. Si la FRA a bien sûr le souci 
de promouvoir l'effectivité de la Charte, force est de constater qu'il s'agit d'un instrument largement ornemental pour la majorité des juridictions nationales.

Sur un échantillon de 70 décisions nationales ${ }^{38}$, la moitié des références à la Charte seulement ont été faites à l'initiative des différentes cours; cette proportion reste globalement stable depuis quelques années ${ }^{39}$. L'utilisation de la Charte varie par ailleurs de façon importante suivant les juridictions. Les cours constitutionnelles ne font que rarement références à la Charte ${ }^{40}$ et l'utilisent encore moins pour juger une législation inconventionnelle, sauf dans certaines situations longuement explicitées par la FRA ${ }^{41}$. La place de la Charte dans les processus législatifs (études d'impact, débats parlementaires) des différents États-membres est encore plus ténue. Même lorsque la norme débattue ou évaluée entre dans le champ du droit de l'Union, les références à la Charte sont peu fréquentes et éclipsées par les citations de la Convention européenne de sauvegarde des droits de l'homme et des libertés fondamentales (Convention EDH) ${ }^{42}$. Celle-ci jouit de toute évidence d'un poids politique nettement plus important.

Lorsque la Charte est utilisée, elle l'est à des fins inappropriées. Comme chaque année, la FRA souligne ainsi la méconnaissance apparente des juridictions du champ recouvert par l'article 51 de la Charte ${ }^{43}$. Ainsi, dans la très grande majorité des décisions analysées, les juges ne se préoccupent pas de montrer que la Charte s'applique bien en l'espèce. Ce n'est que pour écarter un moyen issu de la Charte des droits fondamentaux que les cours mentionnent l'article 51 § 1 de façon explicite. Cela peut s'expliquer en grande partie par la dimension ornementale des utilisations qui sont faites de la Charte. Elle est en effet souvent citée aux côtés d'autres textes conventionnels. Sur les 70 décisions étudiées, 41 d'entre elles mentionnent également la Convention $\mathrm{EDH}^{44}$ et la quasi-totalité sollicitent au moins un autre instrument de protection des droits de l'Homme. La Charte semble ainsi être avant tout un instrument subsidiaire pour les juridictions nationales. Remarquons quoi qu'il en soit que les moyens d'investigation de la FRA sont réduits. En effet, comme elle le souligne, l'utilisation quotidienne de la Charte par les juridictions nationales demeure une « boite noire $»^{45}$ qu'il est difficile de pénétrer. Cela doit conduire à relativiser la portée de son expertise, qui ne porte que sur un échantillon assez modeste.

La portée limitée - soulignée maintes fois - de la Charte européenne des droits fondamentaux aurait pu conduire la FRA à revenir sur le manque d'articulation entre les deux principaux instruments européens de protection des droits de l'Homme. Ce rapport 2017, qui se veut être un bilan des dix dernières années, s'y prêtait idéalement. Or, il est surprenant de constater que l'Agence ne mentionne qu'à peine la question de l'adhésion de l'Union à la Convention. Cette dernière semblait pourtant constituer l'horizon immédiat de la protection des droits fondamentaux au sein de l'UE jusqu'à l'avis $2 / 13^{46} \mathrm{de}$ la Cour de justice de l'Union européenne (CJUE). Un unique paragraphe souligne ainsi le caractère problématique de l'absence de contrôle externe du droit de l'Union ${ }^{47}$.

31 Il aurait sans doute été pertinent et souhaitable de revenir sur l'histoire et les enjeux liés à l'adhésion de l'Union à la Convention EDH (depuis l'avis 2/94). Une courte analyse de l'avis 2/13 aurait trouvé toute sa place dans un rapport se voulant être une rétrospective de l'évolution de la protection des droits fondamentaux, ainsi que des perspectives qu'il ouvre pour le futur. Ceci est d'autant plus vrai qu'il demeure particulièrement critiquable pour une large partie de la doctrine ${ }^{48}$. L'agence se contente de souligner que la nonadhésion pourrait entraîner un problème de perception lié au fait que l'autonomie du droit de l'Union semble primer sur la protection des droits fondamentaux ${ }^{49}$. La frilosité du 
rapport sur cette question peut sans doute être lue comme une manifestation supplémentaire de la position défensive adoptée par l'UE vis-à-vis de la Cour EDH.

Face aux limites affichées par les instruments formels de protection des droits de l'Homme (particulièrement de la Charte), la FRA invite à renforcer la communication autour des droits et libertés au sein de l'espace européen. C'est, au fond, l'un des principaux messages que l'Agence souhaite transmettre à l'aide de ce rapport 2017 : sans le développement d'une réelle culture des droits de l'Homme, la Charte et la Convention EDH ne sauraient avoir qu'une efficacité relative. La FRA invite de la sorte à se méfier des constructions techniques déconnectées de la société civile, n'hésitant pas à imputer la montée des populismes au manque de liens avec les citoyens de l'Union dans le domaine des droits fondamentaux ${ }^{50}$. Elle plaide pour un renouvellement et un renforcement des actions de communication afin de mieux faire face aux critiques, ce qui passe par une meilleure utilisation des relais que constituent les associations. Le financement d'études portant sur les violations des droits de l'Homme et leur diffusion à grande échelle est également un instrument essentiel afin de permettre une plus grande prise de conscience des populations.

La FRA souligne ainsi le caractère nécessaire de son action, avec une forme d'autopromotion. Entre autres, elle mentionne notamment l'impact important de son enquête sur les violences faites aux femmes à l'intérieur de l'Union ${ }^{51}$. Cette mise en avant de l'aspect communicationnel témoigne en creux de l'impuissance d'une institution qui peine à se positionner à la fois à l'intérieur de l'Union et à l'extérieur de celle-ci, comme démontré supra. À défaut de pouvoir jouer un rôle de coordination efficace, tout particulièrement entre l'UE et le Conseil de l'Europe, ou de surveillance efficace à l'égard des États membres, elle semble se rabattre sur une fonction de communication limitée.

\section{B/ - Quelle place pour les droits fondamentaux dans le projet européen?}

Ce rôle de communication nous amène tout d'abord à réinterroger la relation de la FRA avec le Conseil de l'Europe, organisation régionale chargée de la promotion d'une culture des droits de l'Homme. Le rapport ne procède pas à l'analyse des difficultés d'articulation des systèmes de protection des droits fondamentaux de Luxembourg et de Strasbourg, difficultés réactualisées par l'arrêt de la CJUE. Or, l'Agence en elle-même est au cœur de cette problématique. En effet, depuis que l'UE se positionne comme un acteur de premier plan en matière de droits fondamentaux, la FRA a représenté à la fois le symbole de la concurrence entre les deux systèmes et l'espoir d'une coordination possible ${ }^{52}$.

La première décennie des années 2000 consacre une montée en puissance de l'affirmation des droits fondamentaux par l'Union européenne. Cette affirmation se traduit concrètement par l'adoption de la Charte en 2000 et par la création de l'Agence en 2007. Le Traité de Lisbonne entré en vigueur en 2009 en constitue l'apogée, notamment car il rend la Charte contraignante et prévoit l'adhésion de l'UE à la Convention EDH.

C'est dans ce contexte qu'a émergé la problématique de la concurrence avec le système de Strasbourg. La montée en puissance de l'UE en matière de droits fondamentaux est en effet perçue au début des années 2000 comme un risque d'affaiblissement du Conseil de l'Europe ${ }^{53}$. Comme le constatait Jean Petaux en 2009 dans son ouvrage dédié au Conseil de l'Europe, à l'occasion du 60ème anniversaire de l'organisation, L'Europe de la démocratie et 
des Droits de l'homme, le Conseil de l'Europe était passé en vingt ans des "Dix Glorieuses ", les années 90, aux "Dix Grincheuses », les années 2000. Face à ces craintes, la question de la coopération institutionnelle entre l'UE et le Conseil de l'Europe s'est rapidement posée ${ }^{54}$. En 2007, la coopération a été institutionnalisée par un mémorandum d'accord entre l'UE et le Conseil de l'Europe qui faisait suite au rapport de Jean-Claude Juncker «Conseil de l'Europe - Union européenne : une même ambition pour le continent européen ", paru le 11 avril 200655. L'Agence qui est chargée de coopérer avec le Conseil de l'Europe s'inscrit dans cette perspective, tout comme le Traité de Lisbonne qui prévoit l'adhésion de l'UE à la Convention EDH. L'avis de la CJUE est clairement venu mettre un terme à ce processus. Dès lors, en mettant en avant son rôle de communication et de promotion d'une culture des droits de l'Homme, domaine de prédilection du Conseil de l'Europe, il est possible se demander si l'Agence ne participe pas à alimenter ce risque, sinon de concurrence, au moins de redondance entre les deux systèmes.

En creux, il est également possible de se demander si les préconisations du rapport ne reflètent pas la place que les droits fondamentaux occupent actuellement dans la construction européenne. L'hypothèse est que la deuxième décennie des années 2000 est caractérisée par le recul des droits fondamentaux au niveau européen. La lecture du rapport annuel témoigne en effet d'une reconnaissance du mauvais état de santé du système de l'UE en matière de droits de l'Homme. Le bilan décennal est explicitement l'occasion pour la FRA de constater des «droits fondamentaux sous pression " à travers un état des lieux dans quatre domaines clés : la violence envers les femmes, la tension entre protection de la vie privée et la garantie de la sécurité, l'expérience par les Roms de la pauvreté et de la discrimination et, enfin, la situation des enfants migrants. Au-delà de ces quatre domaines mentionnés explicitement, l'analyse des conséquences de l'avis de la CJUE ainsi que le choix de l'utilisation des outils de l'Union économique pour répondre aux problématiques liées aux droits de l'Homme s'apparentent à autant de symptômes d'une Union européenne des droits fondamentaux en perte de vitesse.

L'avis 2/13 de la CJUE a un impact non négligeable sur l'Union européenne des droits fondamentaux. À cet égard, il est intéressant de constater que la Cour de justice s'inscrit ici dans un mouvement de "constitutionnalisation" des droits fondamentaux en s'octroyant un rôle de Cour constitutionnelle. Ce processus n'est pas propre à l'Union européenne. Toutefois, de manière assez paradoxale si dans certains cas cette position de la CJUE s'opère à la faveur des droits fondamentaux, mis en avant par ailleurs dans l'arrêt Safe Harbour, elle peut aussi se retourner contre eux ${ }^{56}$. Dans le cas de l'adhésion de l'UE à la Convention EDH, la CJUE privilégie ainsi clairement l'autonomie du droit de l'UE sur la cohérence du système de protection des droits et libertés fondamentaux.

De quels moyens dispose alors l'UE pour s'affirmer comme acteur de la protection des droits et libertés? Dans le chapitre sur le droit des enfants, l'objectif de réduction de la pauvreté des enfants dans le cadre de la Stratégie UE2020 apparaît en premier. L'agence invite à mobiliser les outils existant au niveau de l'UE pour atteindre cet objectif, en citant en premier lieu le Semestre européen, un outil de finances publiques qui vise à réduire le déficit et la dette publique. Viennent ensuite le pilier européen des droits sociaux, actuellement en négociation, puis l'implication du secteur privé avec les partenariats publics-privés, mentionnés en tant que bonnes pratiques. Ces outils, créés au service de l'intégration économique, reflètent le positionnement ambigu de l'UE face aux droits de l'Homme. Alors même que la protection des droits n'est pas la fonction première de la construction européenne telle qu'elle s'est faite dans le cadre des communautés, 
l'UE dispose pourtant aujourd'hui d'outils plus puissants qu'une organisation internationale classique pour les faire respecter.

Mais le rapport de la FRA le souligne, ces outils ne sont pas forcément aisés à utiliser. En attestent le blocage des négociations depuis plus de 8 ans sur la directive relative aux discriminations ainsi que les difficultés entourant la mise en œuvre de la procédure de l'article 7 TUE. Dans ce dernier cas, l'attitude de la Pologne, qui n'hésite pas à dénoncer les recommandations de la Commission européenne, illustre la dégradation de la capacité de l'UE à s'affirmer comme acteur de promotion et de garantie des droits, malgré les avancées du Traité de Lisbonne en la matière. Enfin, la partie du rapport relative à l'asile, aux visas, à la migration, aux frontières et à l'intégration est relativement limitée. Cela peut s'expliquer par le fait que le focus du rapport annuel de l'année précédente était entièrement consacré à la crise migratoire. Trois recommandations sur six concernent l'éducation des enfants migrants ${ }^{57}$. La FRA interpelle donc la Commission européenne sur la nécessité d'un nouveau plan d'action de l'UE sur les enfants migrants. Or, à l'heure actuelle, si un tel plan a été adopté par le Conseil de l'Europe en mai 2017, il n'y a toujours pas eu d'avancées significatives au niveau de l'UE.

Si le rapport évoque bien des «droits fondamentaux sous pression » dans l'UE, il ne se prête pas à une analyse de plus long terme qui permettrait de mettre en évidence un recul de la place des droits fondamentaux au sein de l'Union européenne au regard de la décennie précédente. L'exercice du rapport annuel en lui-même limite une telle analyse. Toutefois, celle-ci aurait pu trouver sa place dans le cadre du bilan rétrospectif que la FRA a entrepris. On comprend toutefois qu'un tel constat aurait sans doute eu pour conséquence de remettre en question le rôle de l'Agence en tant qu'acteur européen de protection des droits fondamentaux. Actuellement cantonnée dans un rôle d'agence de communication, la FRA laisse néanmoins percevoir son souhait d'apporter une valeur ajoutée en fournissant des "éléments de preuve concernant des violations des droits fondamentaux $\|^{58}$ à l'image de ses homologues nationaux et internationaux. Les rapports à venir permettront de mesurer la réalisation de l'objectif affiché.

Agence des droits fondamentaux de l'Union européenne (FRA), Rapport 2017 sur les droits fondamentaux 2017, Luxembourg : Office des publications de l'UE, 2017

Les Lettres « Actualités Droits-Libertés » (ADL) du CREDOF (pour s'y abonner) sont accessibles sur le site de la Revue des Droits de l'Homme (RevDH) - Contact 


\section{NOTES}

1. Agence des droits fondamentaux de l'Union européenne (FRA), Rapport sur les droits fondamentaux 2017, Luxembourg: Office des publications de l'UE, 2017, p. 22 (ci-après Rapport FRA 2017).

2. Règlement $\mathrm{CE} \mathrm{n}^{\circ} 168 / 2007$ du Conseil du 15 févr. 2007 portant création d'une Agence des droits fondamentaux de l'Union européenne.

3. Elle n'est pas une agence "exécutive» ayant une mission de gestion d'un programme de l'Union.

4. Le Traité de Lisbonne, qui entre en vigueur le $1^{\text {er }}$ décembre 2009, donne à la Charte des droits fondamentaux une valeur juridique égale à celle des traités - Art 6 TUE.

5. Le Conseil d'administration est composé non de représentants des États membres mais d'experts indépendants des droits de l'Homme (art. 12 du règlement $n^{\circ} 168 / 2007$ ). La présence d'un comité scientifique, "organe que l'on retrouve rarement dans les autres agences exécutives de l'Union» (C. Gauthier, S. Platon et D. Szymczak, Droit européen des droits de l'Homme, 2016, p. 436), renforce la crédibilité de son expertise.

6. "The negotiation and framing of FRA's mandate reflected Member States' reticence to create a fullyfledged human rights institution at EU level » (Rapport FRA 2017, op. cit., p. 10).

7. Art $4 \S 2$ du règlement $n^{\circ} 168 / 2007$.

8. Le thème «focus » retenu par la FRA cette année intitulé « Entre promesses et réalisations : 10 ans de droits fondamentaux dans l'UE », explore ces défis et passe en revue les points forts et les lacunes persistantes des 10 dernières années.

9. Cette ambiguïé de positionnement apparent de la FRA a fait l'objet de plusieurs analyses. En 2011, Bal Sokhi-Bulley l'analysait comme le reflet d'un mode de gouvernance particulier, celui d'une surveillance de type panoptique, au sens développé par Foucault dans Surveiller et punir pour expliquer la société disciplinaire. Voir Bal Sokhi-Bulley, “The Fundamental Rights Agency of the European Union: A New Panopticism", Human Rights Law Review, 2011, Oxford University Press. Deux ans plus tard, en 2013, Jahed Morad qui examinait comment la FRA répondait aux lacunes en termes de cohérence du système de protection des droits fondamentaux dans l'UE, mettait en lumière la multiplicité des fonctions de la FRA, incluant la surveillance, mais insistait surtout sur son objectif de stratégie de communication autour des droits de l'Homme. Jahed Morad, "Does the Fundamental Rights Agency cover the gap in monitoring compliance with human rights in the European Union?", The journal of the law society of Scotland, 2013.

10. Règlement $n^{\circ} 168 / 2007$ : Les conclusions, avis et rapports visés au paragraphe 1 excluent les travaux de la Commission dans le cadre de la procédure de do-décision (art. 250 TFFUE), des questions posées à la Commission (article 230 TFUE) et des commissions d'enquête du Parlement européen (article 226 TFUE).

11. Rapport FRA 2017, op. cit., pp. 201-202.

12. De nombreuses manifestations ont eu lieu suite aux déclarations du gouvernement au regard de la Convention d'Istanbul (Convention du Conseil de l'Europe sur la prévention et la lutte contre la violence à l'égard des femmes et la violence domestique).

13. Rapport FRA 2017, op. cit., p. 210.

14. Par exemple, les Pays-Bas sont cités à deux reprises dans la partie traitant du système judiciaire pour mineurs. V. Rapport FRA 2017, op. cit., pp. 180-181.

15. Rapport FRA 2017, op. cit., pp. 203-204.

16. Ibid., pp. 63-64. 
17. L'objectif étant justement de les infléchir pour y inclure la question de la pauvreté des enfants. C'est le cas lorsqu'elle aborde le Semestre européen ou encore le socle européen des droits sociaux dans le cadre des droits de l'enfant, que la Commission souhaite voir adopter rapidement par les co-législateurs. V. Avis de la FRA accompagnant le rapport annuel 2017, p. 19 : recommandation 7.1 de la FRA «l'UE devrait insister davantage sur une réponse globale au problème de la pauvreté et de l'exclusion sociale des enfants dans le cadre du Semestre européen (en utilisant mieux la recommandation de la Commission européenne de 2013) ainsi que dans les initiatives à venir, telles que le pilier européen des droit sociaux ".

18. Le plan d'action sur la protection des enfants réfugiés et migrants en Europe (2017-2019) a été adopté au niveau du Conseil de l'Europe en mai 2017, mais toujours pas au niveau de l'UE. Cf. Partie II-B.

19. À titre d'exemple, est citée la résolution du PE de 2016 appelant à la mise en œuvre d'un mécanisme permanent sur la démocratie, l'État de droit et les droits fondamentaux. (p. $202 \mathrm{du}$ rapport).

20. Le Parlement européen publie chaque année un rapport d'initiative sur la situation des droits fondamentaux dans l'UE.

21. Le Parlement européen a développé une véritable diplomatie des Droits de l'Homme avec une sous-commission dédiée (commission DROI), de nombreuses délégations, des missions d'observation électorale ou encore le Prix Sakharov.

22. Parlement européen, Rapport du 31 mars 2017 concernant la décharge sur l'exécution du budget de l'Agence des droits fondamentaux de l'Union européenne pour l'exercice 2015 (\$5 de l'avis de la commission LIBE).

23. Ibid.

24. En termes de procédure, le Conseil adopte par décision le Cadre pluriannuel de l'Agence, budget qui est ensuite approuvé par le PE. Cf. projet de décision du Conseil établissant un cadre pluriannuel pour l'Agence des droits fondamentaux de l'Union européenne pour la période 2018-2022 (14423/2016 - C8-0528/2016 - 2016/0204(APP)).

25. Dans la première partie du rapport sur le bilan décennal elle mentionne ce rôle lorsqu'elle souligne à travers quatre domaines les lacunes subsistantes dans la protection des droits fondamentaux de l'UE : «Dans chaque domaine, les travaux de la FRA apportent une valeur ajoutée en fournissant des éléments de preuve concernant des violations graves et persistantes des droits fondamentaux ». Cf. Rapport FRA 2017, «Entre promesses et réalisations », op. cit., p. 13.

26. Règlement (CE) n 168/2007 du Conseil du 15 février 2007 portant création d'une Agence des droits fondamentaux de l'Union européenne, op. cit.

27. Article 8 du Règlement (CE) n 168/2007 : "Coopération avec les organisations au niveau des États membres et au niveau international, 1. Aux fins d'assurer une coopération étroite entre l'Agence et les États membres, chaque État membre désigne un fonctionnaire comme agent de liaison national destiné à être le principal correspondant de l'Agence au sein de l'État membre. Les agents de liaison nationaux peuvent notamment soumettre au directeur des avis sur le projet de programme de travail annuel avant que celui-ci soit transmis au conseil d'administration. L'Agence communique aux agents de liaison nationaux tous les documents élaborés conformément à l'article 4, paragraphe 1, points a) à h). 2. Aux fins de l'exécution de ses tâches, l'Agence coopère Avec: a) les organisations gouvernementales et les organes publics compétents en matière de droits fondamentaux dans les États membres, $y$ compris les institutions nationales de défense des droits de l'homme, et b) l'OSCE, tout particulièrement le Bureau des institutions démocratiques et des droits de l'homme (BIDDH), les Nations unies et d'autres organisations internationales. 3. Les modalités administratives de la coopération prévue au paragraphe 2 respectent le droit communautaire".

28. Article 18 Règlement (CE) nº 168/2007 du Conseil du 15 février 2007 : «L'Agence devrait coopérer étroitement avec le Conseil de l'Europe. Cette coopération devrait permettre d'éviter tout chevauchement entre les activités de l'Agence et celles du Conseil de l'Europe, notamment par la mise en place de mécanismes générateurs de complémentarité et de valeur ajoutée, comme la conclusion d'un accord de coopération bilatéral et la participation aux structures de gestion de 
l'Agence d'une personnalité indépendante désignée par le Conseil de l'Europe et dotée du droit de vote approprié. »

29. Article 9 du Chapitre 2 Méthode de travail et coopération du Règlement $n^{\circ} 168 / 2007$ «(...) À cette fin, la Communauté, conformément à la procédure décrite à l'article 300 du traité, conclut un accord avec le Conseil de l'Europe en vue d'instaurer une coopération étroite entre celui-ci et l'Agence. Cet accord comprend également la désignation par le Conseil de l'Europe d'une personnalité indépendante appelée à siéger au conseil d'administration de l'Agence et à son bureau exécutif, conformément aux articles 12 et 13 "

30. Pour le rapport de la FRA au Conseil de l'Europe, voir la partie II. A.

31. Le Défenseur des droits est en effet une autorité constitutionnelle indépendante qui peut formuler des recommandations, prononcer une injonction si ses recommandations ne sont pas suivies d'effet, proposer une transaction, être entendu par toute juridiction à l'initiative de celleci ou à sa demande, saisir le Conseil d'État d'une demande d'avis, etc. À noter qu'il n'hésite pas à mettre en avant sa coopération avec l'Agence sur son site internet. Cependant, ces deux institutions sont difficilement comparables dans le sens où le Défenseur des droits rassemblerait au niveau de l'UE les fonctions de la FRA et du médiateur européen.

32. $\$ 11$ de l'Observation générale $n^{\circ} 33$ du Comité des droits de l'Homme, «Les obligations des États parties en vertu $d u$ Protocole facultatif se rapportant au Pacte international relatif aux droits civils et politiques » 25 juin 2009, 94ème session, CCPR/C/GC/33

33. Ibid. L'observation générale $n^{\circ} 33$ traduit l'interprétation pratique du rôle attribué au comité des droits de l'Homme par ce dernier.

34. La montée en puissance du Parlement européen sur la question des droits fondamentaux en dépit de ses compétences ou le cas du Comité des droits de l'Homme précédemment évoqués sont des exemples de ce type de pratique.

35. L'observatoire était né d'une décision du Conseil de l'Union européenne de 1997.

36. La Convention des Nations Unies relative aux droits des personnes handicapées est la première convention relative aux droits de l'homme à laquelle l'UE est partie.

37. Voir sur le sujet: Jahed Morad, «UE Fundamental Rights Agency : the missing link? ", "The FRA's independance », 14 octobre 2013, The Journal of the law society of Scotland, op. cit.

38. Rapport FRA 2017, op. cit., p. 38.

39. Voir les rapports 2014, 2015.

40. Par exemple, en Lituanie, la cour constitutionnelle n'a fait référence à la Charte qu'une seule fois, contre 178 fois pour la juridiction administrative suprême.

41. Rapport FRA 2017, op. cit., p. 43.

42. "However, such procedures tend not to refer to EU law or the Charter" (Rapport FRA 2017, op. cit., p. 46).

43. Article $51 \S 1$ «Les dispositions de la présente Charte s'adressent aux institutions, organes et organismes de l'Union dans le respect du principe de subsidiarité, ainsi qu'aux États membres uniquement lorsqu'ils mettent en cuvre le droit de l'Union".

44. Rapport FRA 2017, op. cit., p. 39.

45. Ibid., p. 40.

46. CJUE, Ass. plen., Avis 2/13, 18 décembre 2014.

47. Rapport FRA 2017, op. cit., p. 23.

48. E.g. http://www.gdr-elsj.eu/2015/01/31/droits-fondamentaux/3742/.

49. Rapport FRA 2017, op. cit., p. 23.

50. "It is this failure that at least partly explains the rise in support for populist groups in many places throughout Europe" (Rapport FRA 2017, op. cit., p. 22).

51. Publiée en 2014, cette étude estimait que 13 millions de femmes avaient été victimes de violence dans les 12 derniers mois au sein de l'Union européenne. 
52. «La création de l'Agence des droits fondamentaux de l'Union européenne : coopération ou compétition entre le Conseil de l'Europe et l'Union européenne dans le domaine de la protection des droits de l'Homme?» (Jean Philippe Dunand et Pascal Mahon (dir.), "Le droit décloisonné", interférences et interdépendances entre droit privé et droit public, Genève [etc.] : Schulthess, éd. romandes, 2009, pp. 129-148).

53. Les deux organisations ne sont pas dotées du tout du même budget. En 2009, le budget du conseil de l'Europe s'élève à 205 millions d'euros, et celui de l'UE à 11685 milliards d'euros. La montée en puissance de l'UE sur les droits et libertés fondamentaux avait de quoi effrayer le Conseil de l'Europe. A noter toutefois que le budget de la FRA est plutôt de l'ordre de 20 millions d'euros.

54. En témoigne la déclaration de Jean-Claude Juncker, alors premier ministre du Luxembourg et président du Conseil européen lors du sommet des chefs d'Etats des gouvernements organisé à Varsovie en 2005 : «Quand il s'agit de l'essentiel, les rivalités entre le Conseil de l'Union européenne n'ont pas lieu d'être; la sauvegarde des droits de l'homme, l'action culturelle, ainsi que son activité normative font du Conseil de l'Europe une organisation dont l'UE serait dans l'impossibilité de prendre le relais. Le Conseil de l'Europe et l'UE sont d'une grande complémentarité dans leurs domaines d'action et leurs expériences ».

55. Dès la fin des années 80 , il existait une procédure qui consistait dans la correspondance entre le Secrétaire Général du Conseil de l'Europe et le Président de la Commission européenne, à travers la rédaction de lettres d'intentions. Le 3 avril 2001 «la déclaration conjointe sur la coopération et le partenariat entre l'UE et le Conseil de l'Europe », venait formaliser cette pratique. Le traité modifié instituant les Communautés européennes, signé à Amsterdam en 1997 et entré en vigueur en 1999, ouvrait également de nouvelles perspectives de coopération entre l'Union européenne et le Conseil de l'Europe. Le texte du mémorandum est organisé en huit chapitres traitant de la coopération en matière de droits de l'homme, de démocratie, d'État de droit, de la politique européenne de voisinage, du processus de stabilisation et d'association de l'UE, des valeurs européennes sur le terrain (jeunesse, éducation, coopération culturelle, dialogue interculturel), les programmes conjoints (en s'appuyant sur les programmes cités plus haut), les coopérations interinstitutionnelles (avec la question fondamentale de l'adhésion de l'Union européenne au Conseil de l'Europe), et enfin une série de quinze recommandations finales.

56. Grande questions européennes, $4^{\mathrm{e}}$ ed., Horizon, Armand Colin. pp. 205-206: « Dans le dernier quart du XXème siècle, la question de la protection des droits fondamentaux a acquis une dimension constitutionnelle marquée. Le droit de l'Union n'a pas échappé à cette évolution. La CJUE l'a progressivement enrichi. (...) La CJUE a eu l'occasion de renforcer en 2015 son rôle de gardienne des libertés en prononçant l'annulation de l'accord dit "Safe Harbour» signé entre la Commission et les autorités américaines et relatif aux transferts des données personnelles (CJUE, 6 octobre 2015, Schrems). (...) Cette décision très politique, dans laquelle «la Cour s'est arrogé un rôle dans la politique étrangère de l'Union qui ne va pas de soi (B. Hafnel, Dalloz, 2016 p. 111 et s.), assoie un peu plus l'image dotée de pouvoirs constitutionnels à l'instar de l'avis rendu » sur l'adhésion de l'UE à la CESDH".

57. Les autres recommandations concernent le respect de la protection des données dans le cadre des systèmes d'informations relatifs aux migrants, la pratique de la rétention qui devrait être soumise à un objectif légitime, et la promotion des voies légales menant à l'UE notamment pour le regroupement familial des personnes bénéficiant d'une protection internationale.

58. Entre promesses et réalisations : 10 ans de droits fondamentaux dans l'UE, p. 13 


\section{RÉSUMÉS}

Le rapport annuel pour 2017 de l'Agence des droits fondamentaux de l'Union européenne (FRA) dresse un bilan en demi-teinte d'une décennie de promotion des droits et libertés au sein de l'Union. Dans un contexte de crises multiples où les droits fondamentaux sont fragilisés, la FRA plaide pour un engagement et une communication renouvelés autour des droits de l'Homme. Le bilan qui caractérise le rapport constitue une opportunité de s'interroger, par le prisme de l'action de la FRA, sur l'efficacité de la protection des droits fondamentaux au sein de l'UE. Dotée de faibles prérogatives et ballotée entre différents intérêts, la FRA adopte un positionnement ambigu au sein de l'environnement institutionnel de l'Union et collabore avec une multiplicité d'acteurs nationaux, régionaux et internationaux de protection des droits. L'analyse de ces relations montre que, malgré ses prétentions initiales, la FRA se trouve limitée dans son action et ses capacités de surveillance demeurent modestes. Le rôle de communication affiché de l'Agence au service de la promotion d'une culture des droits de l'Homme, en réponse aux limites des instruments formels de l'UE, conduit à réinterroger sa relation avec le Conseil de l'Europe. Plus largement, le positionnement de la FRA dans son rapport reflète en creux la difficulté de l'UE à développer une protection effective des droits fondamentaux.

\section{AUTEURS}

\section{NATHAN DUFROIS}

Étudiant du Master 2 « Droits de l'Homme », Université Paris Nanterre

AGATHE FADIER

Étudiante du Master 2 « Droits de l'Homme », Université Paris Nanterre

\section{ELISABETH MILLER}

Étudiante du Master 2 « Droits de l'Homme », Université Paris Nanterre 